\title{
In-hospital costs for total hip replacement performed using the supercapsular percutaneously-assisted total hip replacement surgical technique
}

\author{
James Chow ${ }^{1}$ - David A. Fitch ${ }^{2}$
}

Received: 12 July 2016 / Accepted: 17 October 2016 / Published online: 12 November 2016

(C) The Author(s) 2016. This article is published with open access at Springerlink.com

\begin{abstract}
Purpose The supercapsular percutaneously-assisted total hip (SuperPath) surgical technique for total hip replacement (THR) is a tissue-sparing approach that has been shown to improve key variables associated with the economic burden of THR (e.g., length of stay, readmissions). To date, no studies have examined the economic impact of using this technique in the United States. The objective of this study was to compare the in-hospital costs of this technique to all other THRs performed in a large hospital system in the United States.

Methods The costing database for a large hospital system was retrospectively searched for all in-hospital costs associated with primary THRs performed between January 2013 and September 2015. Data for all SuperPath THRs (group A) were compared to that of all other THRs performed at centres within the hospital system (group B).

Results Use of the SuperPath technique resulted in significant overall in-hospital cost reductions of $15.0 \%(p<0.000)$, including reductions in operating room costs of $17.3 \%$ $(p<0.000)$, physical/occupational therapy costs of $26.8 \%$ $(p=0.005)$, and pharmacy costs of $25.3 \%(p<0.000)$. Length of stay (1.2 vs. 2.6 days), transfusion rates (1.9 vs. $15.8 \%)$, and 30-day readmission rates (0.4 vs. $2.9 \%)$ were also lower in group A.
\end{abstract}

James Chow

info@ChowHipAndKnee.com

1 Hedley Orthopaedic Institute, 2122 E. Highland Ave., Ste 300, Phoenix, AZ 85016, USA

2 MicroPort Orthopedics Inc., 5677 Airline Rd., Arlington, TN 38002, USA
Conclusions The use of this tissue-sparing surgical technique resulted in reductions in in-hospital costs, length of stay, and readmissions when compared to all other THRs performed in a large hospital system in the United States.

Keywords Economic outcomes · Supercapsular percutaneously-assisted total hip · Tissue-sparing · Total hip replacement

\section{Introduction}

The supercapsular percutaneously-assisted total hip (SuperPath) surgical technique for total hip replacement (THR) is a tissue-sparing approach that utilizes the interval between the piriformis and the gluteus medius to access the hip capsule $[1,2]$. By accessing the hip capsule through this interval, the surgeon is able to preserve musculature and the external rotators vital for allowing early ambulation and reducing the opportunity for post-operative dislocation [3-5]. A recent multicenter study found use of this technique reduced several key factors associated with the economic burden of THR including reductions in length of stay (LOS) of over $50 \%$ (1.6 vs. 3.3 days) and 30-day readmission rates of nearly $2 \%$ ( 2.3 vs. $4.2 \%$ ) when compared to previously reported averages in the United States [6]. Another study showed reductions of in-hospital costs of over $28 \%$ at a centre in Canada when using SuperPath compared to the Hardinge approach [7]. While these reports suggest in-hospital costs could be reduced in the United States using this technique, there have yet to be any studies to confirm. The purpose of the current study was to compare the economic burden of this surgical technique to that of all other THRs performed in a large hospital system based in the United States. 


\section{Methods}

The costing database for a large hospital system was retrospectively searched for all in-hospital costs associated with primary THRs performed between January 2013 and September 2015. Data was compiled in two groups. Group A consisted of all procedures performed by a single surgeon using the SuperPath technique and group B included all other THRs performed within the same hospital system. Group B included data for 34 surgeons at nine hospitals in four states. THRs were included in the analyses if they had an ICD-9-CM primary procedure code of 81.51 , an MS-DRG code of 470 , and primary ICD-9-CM diagnosis codes of 715.15, 715.25, or 715.35. These criteria were selected to ensure the two groups were similar in diagnosis and disease severity, as group A only had two cases with an MS-DRG code different than 470 and only five cases with a diagnosis code different from those stated.

Only costs incurred by the hospital were collected and not charges or reimbursement values. Costing information was collected related to all aspects of the primary in-hospital stay including: anesthesia; intensive care unit; imaging; labs; operating room (OR) time; pharmacy; recovery room; patient room and board; physical/occupational therapy; and transfusions. Patient LOS, transfusion rate, redamission rate, and discharge status were also collected. LOS was defined as the number of nights a patient stayed in the hospital. The transfusion rate was described as the percentage of patients requiring a transfusion. Readmission rate was the percentage of patients readmitted to the hospital for any reason within 30 days following discharge. Discharge status indicated the patient disposition (e.g., routinely home, skilled nursing facility).

\section{Statistical methods}

Age and LOS were presented as means and ranges. Transfusion rate, readmission rate, and discharge statuses were all presented as percentage of the total number of patients in each group. The mean per patient category costs for each group and the percent difference between the groups were calculated. Percent differences were used instead of actual costs to protect proprietary costing information for the hospital system. When appropriate, a t-test $(\alpha=0.05)$ was used to compare per patient costs between the two groups.

\section{Results}

\section{Patient population}

A total of 419 group A and 1673 group B THRs fulfilled the inclusion criteria (Table 1). Group A was younger (61.5 years vs. 65.1 years, $p<0.000$ ) and had a higher percentage of male patients (47.4 vs. $43.9 \%$ ). Patients in group A experienced a $54 \%$ reduction in LOS (1.23 vs. 2.68 days, $p<0.000)$ when compared to group B and over $61 \%$ more where discharged routinely home (91.1 vs. $29.6 \%)$. Operating room $(p=0.004)$ and anesthesia time $(p=0.002)$ were both significantly reduced in group A.
Table 1 Patient demographics, LOS, transfusion rate, readmission rate, and discharge status for the two study groups

\begin{tabular}{lll}
\hline & Group A & Group B \\
\hline N patients & 419 & 1673 \\
Mean age (years)* & 61.5 (range, 26-90) & 65.1 (range, 20-90) \\
Males/females & $199 / 220$ & $735 / 938$ \\
Operating room time (mins)* & 142.7 (range, $88-322)$ & $148.1($ range, $62-430)$ \\
Anesthesia time (mins)* & $142.5($ range, $88-274)$ & $148.7($ range, $62-430)$ \\
Length of stay (days)* & $1.23($ range, $0.5-4.7)$ & $2.68($ range, $0.0-17.2)$ \\
Transfusions (\%) & $8(1.9 \%)$ & $265(15.8 \%)$ \\
$30-$ day readmissions $(\%)$ & $2(0.4 \%)$ & $50(2.9 \%)$ \\
Discharge status & & \\
Home or self care & $382(91.1 \%)$ & $496(29.6 \%)$ \\
SNF & $7(1.6 \%)$ & $349(20.8 \%)$ \\
Home health care & $10(2.3 \%)$ & $707(42.2 \%)$ \\
Rehabilitation facility & $4(0.9 \%)$ & $113(6.7 \%)$ \\
Another hospital & $0(0.0 \%)$ & $3(0.17 \%)$ \\
Court/law enforcement & $0(0.0 \%)$ & $3(0.17 \%)$ \\
Hospital in-patient care & $0(0.0 \%)$ & $1(0.05 \%)$ \\
Death & $0(0.0 \%)$ & $1(0.05 \%)$ \\
\hline
\end{tabular}

*significant difference $(p<0.05)$ 


\section{In-hospital costs}

Overall per patient costs were $15.0 \%$ higher in group B. Table 2 shows the percent difference in per patient costs for each individual cost category. Group B was significantly more costly than group A in all categories except for recovery room, laboratory, ICU, and implant costs. Pharmacy costs were $25.3 \%$ higher in group B. When only costs associated with opioids/opiates were analyzed, group B THRs incurred $49.2 \%$ more costs. Implant costs accounted for the largest percent of the in-hospital costs and were not significantly different between the two groups $(p=0.065)$. When implant costs were excluded from the analysis, overall per patient costs were $36.1 \%$ higher in group B.

\section{0-day readmissions}

There were two readmissions $(0.4 \%)$ in group A. The first was a 68 year old female patient readmitted for a periprosthetic fracture. The patient was revised and sent to a rehabilitation facility 2.9 days after surgery. The second readmission was a 50 year old female admitted for psychoses secondary to bipolar disorder. This readmission was not associated with the THR and the patient was discharged to home 4.3 days after readmission.

There were 50 readmissions $(2.9 \%)$ in group B (Table 3). The most common reasons for readmission were infection $(1.31 \%)$ and periprosthetic fracture $(0.53 \%)$. There were 30 females and 20 males readmitted, which aligned with the overall gender distribution of group B. The mean LOS for readmissions in group $\mathrm{B}$ was 4.28 days (range, 0.6-25.0)

Table 2 Per patient cost comparison between two groups

\begin{tabular}{lll}
\hline Cost category & Per patient percent difference & $p$-value \\
\hline Overall costs & Group B $+15.0 \%$ & $<0.000^{*}$ \\
Implants & Group A +2.8 \% & 0.065 \\
Costs excluding implants & Group B $+36.1 \%$ & $<0.000^{*}$ \\
OR room & Group B +17.3\% & $<0.000^{*}$ \\
Anesthesia & Group B +79.4\% & $<0.000^{*}$ \\
Room and board & Group B $+26.4 \%$ & $<0.000^{*}$ \\
Recovery room & Group A $+12.8 \%$ & $<0.000^{*}$ \\
Physical/occupational therapy & Group B $+26.8 \%$ & $0.005^{*}$ \\
Pharmacy & Group B $+25.3 \%$ & $<0.000^{*}$ \\
Opioids & Group B $+49.2 \%$ & $<0.000^{*}$ \\
Imaging & Group B $+23.0 \%$ & $<0.000^{*}$ \\
Laboratory & Group A $+3.9 \%$ & 0.147 \\
ICU & Group B $+45.0 \%$ & 0.322 \\
Transfusions & Group B $+88.2 \%$ & $<0.000^{*}$ \\
\hline
\end{tabular}

*significant difference
Table 3 Reasons for readmission for groups A and B

\begin{tabular}{lll}
\hline Reason for readmission & Group A & Group B \\
\hline Infection & $0(0.00 \%)$ & $22(1.31 \%)$ \\
Periprosthetic fracture & $1(0.23 \%)$ & $9(0.53 \%)$ \\
Hematoma & $0(0.00 \%)$ & $5(0.29 \%)$ \\
Dislocation & $0(0.00 \%)$ & $5(0.29 \%)$ \\
Wound complications & $0(0.00 \%)$ & $2(0.11 \%)$ \\
Other & $0(0.00 \%)$ & $2(0.11 \%)$ \\
Femoral neck fracture & $0(0.00 \%)$ & $1(0.05 \%)$ \\
Fever & $0(0.00 \%)$ & $1(0.05 \%)$ \\
Implant breakage & $0(0.00 \%)$ & $1(0.05 \%)$ \\
Vertigo & $0(0.00 \%)$ & $1(0.05 \%)$ \\
Psychoses & $1(0.23 \%)$ & $0(0.00 \%)$ \\
Cerebral artery occlusion & $0(0.00 \%)$ & $1(0.05 \%)$ \\
\hline
\end{tabular}

and patients were discharged to home (16.0\%), SNF $(34.0 \%)$, home health care $(30.0 \%)$, rehabilitation $(14.0 \%)$, or a long-term hospital $(4.0 \%)$. One patient $(2.0 \%)$ died during their readmission stay.

\section{Discussion}

Results from the current study show use of the SuperPath technique was associated with a reduction in in-hospital costs of $15.1 \%$. The cost difference increased to $36.1 \%$ when implant costs were excluded. This agreed well with a recent study from Canada showing a $28.4 \%$ reduction in in-hospital costs, excluding implants, when using SuperPath compared to the traditional lateral approach [7]. The current study is the first to examine the economic impact of using this technique in the United States.

Anesthesia, operating room, and physical/occupational therapy costs were all significantly higher in group B. The reduction in anesthesia costs seen in group A was possibly due to the procedure not requiring the use of blocks or regional anesthesia, which are commonly used in THR. Operating room cost reductions could be due to the decreased need for surgical assistants compared to traditional THR techniques or the need for expensive specialty tables or apparatuses as required by some techniques (e.g., some variants of the direct anterior approach) $[8$, 9]. The SuperPath technique was performed with a single surgical assistant without the need for any specialty tables or apparatuses in all cases. As mentioned previously, both operating and anesthesia times were reduced approximately six minutes in group A. It is unlikely though, that such a small improvement alone would result in such high cost reductions.

Pharmacy costs were $25.3 \%$ higher in group B and when only opioid costs were examined the difference 
increased to $49.2 \%$. These costs included any drug delivered during the hospital stay. The reduction in pharmacy costs was likely multifactorial. Only oral medications were used in group A patients. No patient-controlled analgesia or boutique medications (e.g., intravenous Tylenol or Exparel) were used in any group A patients. Finally, the use of minimally-invasive or tissue-sparing techniques has been shown to reduce pain levels and the amount of pain medication consumed [10-12]. Therefore, it is likely that the group A patients consumed less pain medication, and in particular powerful opioids, on average due to the sparing of the musculature and surrounding soft tissues.

The transfusion rate in group A was nearly $14 \%$ lower than that of group $\mathrm{B}$, resulting in mean transfusion cost savings of $88.2 \%$. Transfusion rates can be affected by several factors including the amount of soft tissue damage caused by the surgical technique. As previously mentioned, the SuperPath technique does not require the cutting of muscles or tendons by utilizing the interval between the piriformis and the gluteus medius. Another factor affecting transfusion rates is the use of antifibrinolytics. In group A, $95.9 \%$ of patients received aminocaproic acid and the remaining $4.1 \%$ received tranexamic acid. Nearly a third $(33.2 \%)$ of patients in group B received tranexamic acid and $27.7 \%$ received aminocaproic acid. When examining only those patients receiving aminocaproic acid, the transfusion rate was $1.7 \%$ (7 of 402) in group A and $7.4 \%$ (35 of 470) in group B. This suggests the surgical technique and not choice of antifibrinolytics could be responsible for the reduction in the need for transfusions. Only a single study could be found comparing the effectiveness of aminocaproic and tranexamic acid for preventing transfusions following joint replacement [13]. That study found nearly five times (12.5 vs. $2.8 \%)$ as many aminocaproic acid patients required transfusion as did tranexamic acid patients. Interestingly in the current study, $7.4 \%$ (35 of 470) of group B patients receiving aminocaproic acid and $20.5 \%$ (114 of 557) of group B patients receiving tranexamic acid required transfusions.

Over $91 \%$ of patients in group A were routinely discharged home compared with just $29.6 \%$ of group B. While discharge status does not affect in-hospital costs, it does have a potentially significant impact on costs for hospitals participating in bundling. Bozic et al. reported over $35 \%$ of charges associated with a 30-day episode of care were related to post-discharge care, with $70 \%$ of postdischarge payments due to discharging patients to post-acute care facilities [14]. Ramos et al. reported the average costs for discharge to an inpatient rehabilitation facility, skilled nursing facility, and home with care as $\$ 16,464, \$ 6,678$, and $\$ 4,239$, respectively [15]. These studies emphasize the potential significant benefit of reducing inpatient rehabilitation facility discharges from $6.7 \%$ in group B to $0.9 \%$ in group A. Similar to discharge status, readmission rates do not impact in-hospital costs but are significant in a bundling scenario. Bozic et al. also reported the cost burden of unplanned THR readmissions to be $4.3 \%$ and the average costs of each readmission to be $\$ 17,103$ [16]. The readmission rate for group A was over seven times lower than that of group B $(0.4$ vs. $2.9 \%)$ in the current study.

While the current study is a review of data independently collected by a hospital system on a large group of patients, there are several limitations. The two groups had some differences in population characteristics. Group A was nearly four years younger and had $3.5 \%$ more male patients. Efforts were made to control the indications and diagnoses for the procedure during patient selection criteria, but not all aspects could be controlled. There was a single surgeon performing the SuperPath procedure and it is therefore possible that the skill of the surgeon and not the technique itself could bias results. There are multiple surgeons included in group B and there are a number of factors (e.g., surgical technique, implant selection, surgeon skill) that could affect results. There are also multiple hospitals included in group B and therefore differences in conditions or procedures (e.g., indications for transfusion) at each of these centers could affect costs.

A final limitation is that this study does not include the learning curve associated with the SuperPath technique, as the single surgeon had significant previous experience with the technique. Costs during the learning curve with a technique can be higher due to the potential for increased LOS, transfusions, surgical time, complications, and readmissions. The previously mentioned study comparing the SuperPath technique to the traditional lateral included the initial 49 SuperPath surgeries performed by the author [7]. This study found complication rates and associated in-hospital costs were all lower in the SuperPath group even during this learning curve phase.

In conclusion, the use of the SuperPath technique resulted in reductions of in-hospital costs, LOS, and readmissions when compared to all other THRs performed in a large hospital system in the United States. Additionally, over $60 \%$ more patients were discharged directly home. Future studies are needed to determine the economic impact of this technique across an entire 30-day episode of care and to compare to specific surgical techniques.

\section{Compliance with ethical standards}

Conflict of interest One author is an employee of MicroPort Orthopedics Inc. 
Funding There is no funding source.

Ethical approval Ethical approval was obtained prior to conducting this study.

Open Access This article is distributed under the terms of the Creative Commons Attribution 4.0 International License (http:// creativecommons.org/licenses/by/4.0/), which permits unrestricted use, distribution, and reproduction in any medium, provided you give appropriate credit to the original author(s) and the source, provide a link to the Creative Commons license, and indicate if changes were made.

Open Access This article is distributed under the terms of the Creative Commons Attribution 4.0 International License (http:// creativecommons.org/licenses/by/4.0/), which permits unrestricted use, distribution, and reproduction in any medium, provided you give appropriate credit to the original author(s) and the source, provide a link to the Creative Commons license, and indicate if changes were made.

\section{References}

1. Chow J, Penenberg B, Murphy S (2011) Modified micro-superior percutaneously-assisted total hip: early experiences $\&$ case reports. Curr Rev Musculoskelet Med 4(3):146-150. doi:10.1007/s12178011-9090-y

2. Della Torre PK, Fitch DA, Chow JC (2015) Supercapsular percutaneously-assisted total hip arthroplasty: radiographic outcomes and surgical technique. Ann Transl Med 3(13):180. doi:10.3978/j.issn.2305-5839.2015.08.04

3. Prigent F (2008) Incidence of capsular closure and piriformis preservation on the prevention of dislocation after toal hip arthroplasty through the minimal posterior approach: comparative series of 196 patients. Eur J Orthop Surg Traumatol 18(5):333-337

4. Khan RJ, Maor D, Hofmann M, Haebich S (2012) A comparison of a less invasive piriformis-sparing approach versus the standard posterior approach to the hip: a randomised controlled trial. J Bone Joint Surg Br Vol 94(1):43-50. doi:10.1302/0301-620X.94 B1.27001

5. Moussallem CD, Hoyek FA, Lahoud JC (2012) Incidence of piriformis tendon preservation on the dislocation rate of total hip replacement following the posterior approach: a series of 226 cases. Le J Medical Libanais Lebanese Med J 60(1):19-23

6. Gofton W, Chow J, Olsen KD, Fitch DA (2015) Thirty-day readmission rate and discharge status following total hip arthroplasty using the supercapsular percutaneously-assisted total hip surgical technique. Int Orthop 39(5):847-851. doi:10.1007/s00264-014-2587-4

7. Gofton W, Fitch DA (2015) In-hospital cost comparison between the standard lateral and supercapsular percutaneously-assisted total hip surgical techniques for total hip replacement. Int Orthop. doi:10.1007/s00264-015-2878-4

8. Matta JM, Shahrdar C, Ferguson T (2005) Single-incision anterior approach for total hip arthroplasty on an orthopaedic table. Clin Orthop Relat Res 441:115-124

9. Woolson ST, Pouliot MA, Huddleston JI (2009) Primary total hip arthroplasty using an anterior approach and a fracture table: shortterm results from a community hospital. J Arthroplast 24(7):9991005. doi:10.1016/j.arth.2009.04.001

10. Goebel S, Steinert AF, Schillinger J, Eulert J, Broscheit J, Rudert M, Noth U (2012) Reduced postoperative pain in total hip arthroplasty after minimal-invasive anterior approach. Int Orthop 36(3):491498. doi:10.1007/s00264-011-1280-0

11. Dorr LD, Maheshwari AV, Long WT, Wan Z, Sirianni LE (2007) Early pain relief and function after posterior minimally invasive and conventional total hip arthroplasty. A prospective, randomized, blinded study. J Bone Joint Surg Am 89(6):1153-1160. doi:10.2106/JBJS.F.00940

12. Zawadsky MW, Paulus MC, Murray PJ, Johansen MA (2014) Early outcome comparison between the direct anterior approach and the mini-incision posterior approach for primary total hip arthroplasty: 150 consecutive cases. J Arthroplast 29(6):1256-1260. doi:10.1016 /j.arth.2013.11.013

13. Camarasa MA, Olle G, Serra-Prat M, Martin A, Sanchez M, Ricos P, Perez A, Opisso L (2006) Efficacy of aminocaproic, tranexamic acids in the control of bleeding during total knee replacement: a randomized clinical trial. Br J Anaesth 96(5):576-582. doi:10.1093 /bja/ael057

14. Bozic KJ, Ward L, Vail TP, Maze M (2014) Bundled payments in total joint arthroplasty: targeting opportunities for quality improvement and cost reduction. Clin Orthop Relat Res 472(1):188-193. doi:10.1007/s11999-013-3034-3

15. Ramos NL, Wang EL, Karia RJ, Hutzler LH, Lajam CM, Bosco JA (2014) Correlation between physician specific discharge costs, LOS, and 30-day readmission rates: an analysis of 1,831 cases. J Arthroplast 29(9):1717-1722. doi:10.1016/j.arth.2014.04.005

16. Bosco JA 3rd, Karkenny AJ, Hutzler LH, Slover JD, Iorio R (2014) Cost burden of 30-day readmissions following Medicare total hip and knee arthroplasty. J Arthroplast 29(5):903-905. doi:10.1016/j. arth.2013.11.006 